\title{
The Comparison of Sexual Function Before and After Pessary Placement in Women with Pelvic Floor Disorders
}

\author{
Farzane Farhang1, Parvin Bastani² ${ }^{2}$ Zhila Khamnian ${ }^{3}$, Maryam Pourbarghi ${ }^{4}$, Fatemeh Mallah ${ }^{5}$
}

\begin{abstract}
1Women's Reproductive Health Research Center, Tabriz University of Medical Sciences, Tabriz, Iran. ${ }^{2}$ Associate Professor, Fellowship of Urogynecology and Pelvic Reconstructive Surgery, Women's Reproductive Health Research Center, Tabriz University of Medical Sciences, Tabriz, Iran. ${ }^{3}$ Assistant Professor, Department of Community Medicine, Tabriz University of Medical Sciences, Tabriz, Iran. ${ }^{4}$ Women's Reproductive Health Research Center, Tabriz University of Medical Sciences, Tabriz, Iran. ${ }^{5}$ Associate Professor, Fellowship of Pelvic Floor Disorder, Women's Reproductive Health Research Center, Tabriz University of Medical Sciences, Tabriz, Iran.
\end{abstract}

\section{ABSTRACT}

\section{BACKGROUND}

Sexual dysfunction is a common problem in women with pelvic organ prolapse. Sexual dysfunction affects their quality of life as well as sexual intercourse. Thus, the present study aims at investigating the sexual function before and after pessary placement in women suffering from pelvic floor disorders.

\section{METHODS}

The design of the present study was a before-after one. The present study was conducted on 96 more than 50-year-old patients who had pelvic floor disorders and were incapable of make sexual intercourses. The patients had already been referred to Al-Zahra and Taleghani Hospitals in Tabriz between 2016 and 2018. The patients had the indication of using a pessary. According to POP-Q system, the intensity of prolapse was determined and pessary placement was conducted for them. For investigating the sexual function, the researchers applied Pelvic Organ ProlapseUrinary Incontinence Sexual Function Questionnaire. For comparing the qualitative variables, $\mathrm{X}^{2}$ test was used. Data analysis was conducted in SPSS-23. For describing the research units, the researchers used mean, standard deviation, and frequency distribution. In terms of the statistical significance, the $p$ values were determined to be less than 0.05 .

\section{RESULTS}

As many as 27 patients (28.1\%) suffered from apex prolapse. Moreover, 5 patients (5.2\%) suffered from anterior vaginal wall prolapse, 28 patients $(29.2 \%)$ suffered from both apexes prolapse and anterior vaginal wall prolapse, and 36 patients $(37.5 \%)$ suffered from apex prolapse and anterior and posterior vaginal wall prolapse. In 84 patients (87.5\%), the pessary was a ring, and in 12 patients (12.5), the pessary was a Gelhorn. There is a significant relationship between the type of pessary and the sexual function of patients with prolapse $(\mathrm{p}=0.006)$. None of the patients attempted to pull out the pessary during their sexual intercourse.

\section{CONCLUSIONS}

Using pessary has no negative effect on the sexual intercourse. Even in some cases pessary not only treats prolapse and urinary symptoms but also improves the patient's self-image; pessary can improve the sexual function. Moreover, there is a significant relationship between the type of pessary and sexual satisfaction in women suffering from pelvic floor disorders.

\section{KEY WORDS}

Pessary Management, Sexual Function, Complications
Corresponding Author:

Fatemeh Mallah,

Department of Obstetrics and Gynaecology, Women's Reproductive Health Research Center, Tabriz University of Medical Sciences, Tabriz, Iran.

E-mail: fatima_m815@yahoo.com

DOI: $10.14260 /$ jemds/2019/585

Financial or Other Competing Interests: None.

How to Cite This Article:

Farhang F, Bastani P, Khamnian $Z$, et al. The comparison of sexual function before and after pessary placement in women with pelvic floor disorders. J. Evolution Med. Dent. Sci. 2019;8(34):2694-2697, DOI: $10.14260 /$ jemds/2019/585

Submission 27-06-2019,

Peer Review 11-08-2019,

Acceptance 19-08-2019,

Published 26-08-2019.

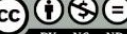




\section{BACKGROUND}

Pelvic floor disorders (PFDs) are one of the most prevalent problems of women all over the world. It has been estimated that nearly $23-50 \%$ of women suffer from pelvic floor disorders in the reproductive age; as many a $3-5 \%$ of these disorders bring about disorders in the patients' lives.(1) The most frequent age for the prevalence of symptoms in patients with pelvic floor disorders is the eighth decade of life.(2) In patients with pelvic floor problems, the sexual problems will increase as well; as many as $11 \%$ of women with pelvic floor disorders complain about sexual problems as well.(3) The sexual function of women is greatly complicated and depends on various factors including stress, depression, anxiety, and infertility. It has been estimated that $38-63 \%$ of women deal with some kind of sexual problem in their lifetime.(4) There is little information about women's pelvic problems and their sexual function. However, it is obvious that in comparison to the ordinary population, women with pelvic floor disorders have more sexual problems. This can be problematic for younger women at the reproductive age.(5)

One of the main treatments of pelvic floor disorders is using pessary that can improve rectocele, cystocele, and urinary incontinence. $(6$ and 7 ) The main problem of using pessary is sexual dysfunction and reduced sexual pleasure, ${ }^{8}$ and 9) and most importantly the unpleasant feeling of the presence of an external object in one's vagina during the sexual intercourse.

Unfortunately, few studies have been conducted on the effect of pessary on sexual intercourse. In the study conducted by Handa V L et al (2011), it was indicated that in comparison to women applying other non-surgical methods, women who use pessaries have more disorders in terms of achieving orgasm and libido. (9) In their study, Abdool Z et al indicated that despite the relative superiority of surgical method to pessary, this superiority is not statistically significant. Moreover, in their study, the sexual intercourse of the group undergoing surgery was more than that of the other group. After conducting the age assimilation, this difference was resolved as well.(10) In another study conducted by Meriwether KV et al (2015), it was indicated that there is no difference in the patients' sexual drive before pessary placement and three months after its placement. However, due to the improvement of pelvic floor disorders in the patients, pessary has increased their sexual function and self-confidence.(11)

Different methods of treating pelvic floor disorders have advantages and disadvantages. Pessary placement is a nonsurgical reversible method that has both an acceptable performance and fewer complications. However, given the insufficient contradictory data on its effect on sexual intercourses, pessary has been commonly treated with scepticism. Thus, the present study was conducted to investigate the effect of pessary on sexual function, the need for pulling out pessary during the sexual intercourse, and the factors affecting the sexual function and body image in women pelvic floor disorders who use pessaries.

\section{METHODS}

The design of the present study was a before-after one. The present study was conducted on patients referring to $\mathrm{Al}$ Zahra and Taleghani hospitals in Tabriz within 2016-2018. The patients had pelvic floor disorders. Moreover, the patients had the indication of using pessary and they were satisfied with using it for treatment. The study was conducted as a clinical trial in two years. The samples were required to have these conditions: having the ability to have sexual intercourse, being older than 50 years old, and not having numerous sexual partners. The sampling method was convenience sampling, and 96 patients entered the study. The individuals who were not able to enter the study include: 1 . Having the history of chronic diseases (Diabetes, high blood pressure (Hypertension), and heart diseases) that affect the sexual function of either of the spouses; 2 . Sexual problems of the spouse such as premature ejaculation and sexual impotence; 3 . The use of drugs affecting the sexual function, narcotics, and alcohol in either of the spouses. The intensity of the prolapse was determined by female attending physicians and residents according to the POP-Q system. Then according to the type of prolapse and its intensity, the size of pessary was determined, and placement was conducted. After the placement of pessary, the visits were conducted one week, two weeks, one month, three months, and six months after the placement. In each visit, the pessary was pulled out, cleaned, and reinserted after the examination and making sure that there is no problem in terms of wound and infection. In the visits conducted two weeks and three months after the pessary placement, Pelvic Organ ProlapseUrinary Incontinence Sexual Function Questionnaire (PISQ12) was used for investigating the sexual function that included 12 questions for evaluating the sexual function of women with prolapses of pelvic organs through behaviouralemotive, physical, and partner-related domains. The validity and reliability of this questionnaire has been already confirmed by Momenimovahed et al (2014). Moreover, the patients were also required to fill the part of the questionnaire dedicated to pessary management during the sexual intercourse. In the aforementioned part, the patients indicated whether they pulled out the pessary and its main cause i.e. personal reasons or upon a request from their sexual partner. For comparing the quantitative variables, $\mathrm{X} 2$ test or Fisher's exact test were used. Data analysis was conducted by using SPSS-23. For describing the research units, the researchers used mean, standard deviation, and frequency distribution. In terms of the statistical significance, the $\mathrm{p}$ values was determined to be less than 0.05 .

\section{Ethical Considerations}

The present study was confirmed by the Ethics Committee of Tabriz University of Medical Sciences. The patients' informed consent was acquired through providing letters of consent as well as the ethical code of Research Deputy of School of Medicine. The patients' information was kept confidential. Moreover, an identification code was allocated for each patient. The project executors were committed to keep the patients' secrets and information. For preserving the confidentiality of the participants' names and addresses, they weren't named, and the data were generally analyzed and interpreted. 


\section{RESULTS}

From among 96 patients, the pessary was pulled out in two cases. One of them pulled out the pessary for vaginal discharge and the other couldn't tolerate it and was not willing to reinsert pessary. The remaining 94 patients did not pull out pessary during their sexual intercourse. In 84 patients $(87.5 \%)$, the pessary was a ring, and in 12 patients (12.5), the pessary was a Gelhorn. The patients' mean age was 68. The mean number of pregnancies was $6.15 \pm 2.70$, and the mean number of deliveries was $5.33 \pm 2.31$. From among 96 patients investigated, as many as 92 patients $(96.2 \%)$ had natural deliveries, and 3 patients $(3.1 \%)$ had experienced both types of deliveries. As many as 27 patients (28.1\%) suffered from apex prolapse. Moreover, 5 patients (5.2\%) suffered from anterior vaginal wall prolapse, 28 patients $(29.2 \%)$ suffered from both apexes prolapse and anterior vaginal wall prolapse, and 36 patients (37.5\%) suffered from apex prolapse and anterior and posterior vaginal wall prolapse. The urinary symptoms were observed in 74 patients $(77.1 \%)$, and 22 patients $(22.9 \%)$ showed no urinary symptoms. From among 74 patients having urinary symptoms, as many as 45 patients $(46.9 \%)$ suffered from urgency incontinence. From among these patients, 41 patients $(42.7 \%)$ were recovered and 4 patients $(4.2 \%)$ were not. As many as 26 patients suffered from stress incontinency. After using the pessary, 24 patients (25\%) were recovered and 2 patients $(2.1 \%)$ were not. As many as 3 patients suffered from nocturia and they were recovered after the treatment.

From among 74 patients having urinary symptoms, 25 patients $(26 \%)$ suffered from urinary retention. After using the pessary, these 25 patients were recovered. As many as 25 patients $(26 \%)$ were complaining about other cases; after the pessary placement, 7 patients (7.3\%) were recovered and 18 patients (18.7) were still complaining about their untreated problems.

The sexual function of 94 patients (Based on the type of pessary used) has been provided in table 1 . According the results of the chi-squared test, there is a significant relationship between the type of pessary and the sexual function of the patients suffering from prolapse $(\mathrm{P}=0.006)$.

\begin{tabular}{|c|c|c|c|c|c|}
\hline \multirow{2}{*}{$\begin{array}{c}\text { Type of } \\
\text { Pessary }\end{array}$} & Satisfaction & Dissatisfaction & No Difference & \multirow{2}{*}{ Total } & \multirow{2}{*}{ p Value } \\
\hline Ring & $36(43.9 \%)$ & $1(1.2 \%)$ & $45(54.9 \%)$ & 82 & \\
\hline Gellhorn & 0 & $11(91.7 \%)$ & $1(50 \%)$ & 12 & \multirow{2}{*}{0.006} \\
\hline Total & $\mathbf{3 6}(\mathbf{3 8 . 3} \%)$ & $\mathbf{1 2}(12.8 \%)$ & $\mathbf{5 1}(54.3 \%)$ & $\mathbf{9 4}$ & \\
\hline \multicolumn{4}{|r|}{ Table 1. The Effect of the Type of Pessary on Sexual Function of } \\
Patients Suffering from Pelvic Flood Disorders \\
\hline
\end{tabular}

\section{DISCUSSION}

One of the most common complaints of patients suffering from pelvic organ prolapse (POP) is sexual dysfunction. Pelvic organ prolapse is the dislocation of a pelvic organ into or out of vagina. Nearly one third of POP patients suffer from sexual dysfunction and dissatisfaction.(1-6) The present study was conducted to investigate the sexual function and pessary management in 96 patients referring to Al-Zahra Hospital and Taleghani hospitals. The mean age of the patients suffering from POP was $68.10 \pm 10.3$ years. The use of pessary is one of the prevalent methods of treating pelvic decent; by keeping the pelvic organs in their right location, it can improve the uterine prolapse, rectocele, cystocele, and urinary incontinence. ${ }^{(7)}$ The studies conducted so far indicate the effectiveness of pessary in treating pelvic disorders and urinary incontinence. However, the main problem of using pessary is the sexual dysfunction and reduced sexual pleasure arising from pessary. In the present study, as many as 74 patients $(77.1 \%)$ were complaining about urinary symptoms including urgency incontinency, stress incontinency, nocturia, and urinary retention before the use of pessary. However, after using pessary, these symptoms were recovered; only $18.3 \%$ of the patients were not recovered. Thus, in the present study, the use of pessary resulted in the improvement of urinary symptoms as well.

In the present study, $37.5 \%$ of the patients were satisfied with their sexual intercourse. As many as $54.3 \%$ of the patients didn't experience any change in their sexual function after the pessary placement. This calls for conducting further studies in this regard.

In the present study, there was a significant relationship between the improved sexual function and the type of pessary used in women suffering from prolapse; using ring pessary had significant effects on the patients' improved sexual function $(9.43 \%)$. The interventions that improve the pelvic floor prolapse improve the sexual function as well; only few patients indicated dissatisfaction with their sexual intercourse $(1.2 \%)$. This is not in line with the findings of the study conducted by Handa et al.(9) In the study conducted by Handa et al, in comparison to women applying non-surgical methods, women using pessary experienced more disorder in achieving orgasm and libido. Moreover, they experienced an unpleasant feeling of an external object in their vagina. ${ }^{(9)}$

However, as many as $91.7 \%$ of the patients using Gelhorn pessary experienced sexual dissatisfaction with their sexual intercourse after the pessary placement. The findings of the present study indicate that using pessary can be considered a non-aggressive medical treatment. Moreover, the type of the pessary used is effective as well. However, further studies are required to be conducted in this regard.

In the study conducted by Abdool et al, the women suffering from pelvic organ prolapse were investigated in two groups: the use of vaginal pessary and surgery. In the oneyear follow-up of the patients, the findings of their study indicated that there was no significant difference between the surgery and pessary groups. (10) The method applied in the present study is different from that of Abdool et al. In their study, they concluded that the patients undergoing surgery were not significantly different from patients using pessary in terms of sexual satisfaction. However, the present study indicated that non-aggressive methods could be also used for treating patients with pelvic floor disorders; in the present study, the use of pessary was followed by sexual satisfaction in $38.3 \%$ of the patients.

Moreover, another study conducted by Abdool $\mathrm{Z}$ et al (2011) was followed by the following results: 554 patients with pelvic organ prolapse were entered into their study. As many as 359 patients underwent pessary treatment and 195 patients underwent surgery. The results of the one-year follow-up indicated that despite the relative superiority of surgery to pessary, this superiority is not statistically 
significant. Moreover, in their study, the sexual intercourse of the group undergoing surgery was more than that of the other group. After conducting the age assimilation, this difference was resolved as well.(10)

\section{CONCLUSIONS}

Using pessary has no negative effect on the sexual intercourse. In some cases, pessary not only treats prolapse and urinary symptoms but also improves the patient's selfimage; pessary can improve the sexual function. Moreover, there is a significant relationship between the type of pessary and sexual satisfaction in women suffering from pelvic floor disorders.

\section{Limitations}

One of the main limitations of the present study was the lack of using different kinds of pessaries; only two types were used in the present study. Another weak point of the present study is different individuals' expectation from the sexual intercourse were not investigated; this can affect the results of the study. Moreover, numerous factors and variables (Biological, cultural, and social) affect the sexual function; controlling all of these factors was not possible in the present study. In addition, given our limited understanding of the physiology of natural sexual function, no objective laboratory method is recommended for evaluating the sexual function; this is a serious limitation of the present study. Last but not least, talking about sexual issues is culturally despicable and heinous in the Iranian society. It is likely that individuals don't feel comfortable talking about their sexual problems both before and after using pessaries.

\section{REFERENCES}

[1] Barber MD, Maher C. Epidemiology and outcome assessment of pelvic organ prolapsed. Int Urogynecol J 2013;24(11):1783-90.
[2] Maher C, Baessler K, Barber M, et al. Surgical management of pelvic organ prolapse. In: International Consultation on Incontinence. Health Publication 2013;7(5).

[3] Simonelli C, Tripodi F, Cosmi V, et al. What do men and women ask a helpline on sexual concerns? Results of an Italian telephone counselling service. Int J Clin Pract 2010;64(3):360-70.

[4] Ozaki Y, Nagao K, Saigo R, et al. Sexual problems among Japanese women: data from an online helpline. Sex Med 2015;3(4):295-301.

[5] Kanter G, Rogers RG, Pauls RN, et al. A strong pelvic floor is associated with higher rates of sexual activity in women with pelvic floor disorders. Int Urogynecol J 2015;26(7):991-6

[6] Kapoor DS, Thakar R, Sultan AH, et al. Conservative versus surgical management of prolapse: What dictates patient choice? Int Urogynecol J Pelvic Floor Dysfunct 2009;20(10):1157-61.

[7] Komesu YM, Rogers RG, Rode MA, et al. Pelvic floor symptom changes in pessary users. Am J Obstet Gynecol 2007;197(6):620.e1-6.

[8] Kenton K, Barber M, Wang L, et al. Pelvic floor symptoms improve similarly after pessary and behavioral treatment for stress incontinence. Female Pelvic Med Reconstr Surg 2012;18(2):118-21.

[9] Handa VL, Dwyer PL. Sexual function and pelvic floor disorders treatment. Best Pract Res Clin Obstet Gynecol 2011;19:993-1008.

[10] Abdool Z, Thakar R, Sultan AH, et al. Prospective evaluation of outcome of vaginal pessaries versus surgery in women with symptomatic pelvic organ prolapsed. Int Urogynecol J 2011;22(3):273-8.

[11] Meriwether KV, Komesu YM, Craig E, et al. Sexual function and pessary management among women using a pessary for pelvic floor disorders. J Sex Med 2015;12(12):2339-49. 